\title{
La Ley Sarbanes-Oxley y su necesidad en las entidades colombianas como solución a los casos de corrupción en temas financieros
}

\author{
Edwin Steven Matallana Chilito ${ }^{1}$ \\ David Leornardo Segura Ávila
}

Recibido: 17 de abril de 2014

Aprobado: 20 de mayo de 2014

\begin{abstract}
Matallana, S. \& Segura, D. (2014). La ley Sarbannes -Oaxley y su necesidad en las entidades colombianas, como solución a los casos de corrupción en temas financieros. Activos 22, 195-228
\end{abstract}

Clasificación JEL: M42

\section{Resumen}

La Ley Sarbanes-Oxley, firmada en Estados Unidos y que rige en este país, tiene gran importancia para las empresas que allí se dirigen por ella para su funcionamiento y credibilidad ante otras entidades, así como para las organizaciones que cotizan en la Bolsa y que son reguladas por la Securities and Exchange Commission $[\mathrm{SEC}]^{3}$.

1 Estudiante del programa Contaduría Pública, Universidad Santo Tomás sede Bogotá. Correo electrónico: edwin.matallana@usantotomas.edu.co

2 Estudiante del programa Contaduría Pública, Universidad Santo Tomás sede Bogotá. Correo electrónico: edwin.matallana@usantotomas.edu.co

3 Comisión de Bolsa y Valores, institución independiente del Gobierno de Estados Unidos, encargada de vigilar el cumplimiento de las leyes federales del mercado de valores, la regulación de la Bolsa de Valores y la información financiera del país. 
A lo largo de este trabajo, se abordará históricamente la estructuración de la Ley Sarbanes-Oaxley (SOX), sus objetivos y la importancia que esta tiene en el contexto norteamericano. Así mismo, se analizarán las estructuras de regulación y las sanciones a entidades y profesionales que no cumplan con los requerimientos establecidos por dicha norma.

El sentido final de este trabajo es analizar si una norma de las características de la SOX es necesaria en el caso colombiano, dado que en nuestro país, los continuos casos de corrupción en empresas en las cuales los profesionales no hacen su trabajo con responsabilidad y ética hacen que se pueda adoptar una ley como la SOX, que regule a todas las empresas de forma estricta. Para esto es necesaria, en primer lugar, la existencia de una comisión que busque el campo de formación para los profesionales, con el fin de adoptar y poner en práctica dicha ley; en segundo lugar, es indispensable que existan sanciones que se apliquen a entidades y profesionales, esto con la finalidad de que no haya escándalos de corrupción y, por lo tanto, no se pierda la confiabilidad en estas entidades que cotizan en la Bolsa y que pertenecen a diferentes industrias.

\section{Palabras clave}

Ley Sarbanes-Oxley, gobierno corporativo, control interno, auditoría, corrupción, contabilidad.

(Moeller, 2004). 


\title{
Matallana, S. \& Segura, D. (2014). The Sarbanes Oxley Act and its need in Colombian institutions as a solution to corruption in financial matters. Activos 22, 195-228
}

\begin{abstract}
The Sarbanes-Oxley Act, signed in the U.S and which is currently in force in this country, is of vital importance for companies which are governed under it, for their credibility and functioning with other entities as well as for those organizations that are listed in the stock exchange market and which are regulated by the Securities and Exchange Commission [SEC].

Throughout this article we will examine the structuring of the Sarbannes-Oaxley (SOX) Act, its aims and the importance it has in the North American context. Similarly we will analyze the regulation structures and the sanctions for professional and entities which do not meet the requirements established by this regulation.

The final aim of this work is to analyze if a SOX law could be applicable in the case of Colombia, since in our country the constant cases of corruption in enterprises in which professionals are neither responsible nor ethical makes it plausible to adopt such a law, which regulates companies strictly. For this it is necessary the creation of a commission which investigates how to train professionals in order to adopt and put this law in practice; secondly, it is essential to have sanctions that can be given to entities and professionals in order to prevent corruption scandals, thus, protecting confidence in these entities listed in the stock exchange market and which belong to different industries.
\end{abstract}

\section{Keywords}

Sarbanes-Oxley Act, corporate governance, internal control, auditing, corruption, accounting. 
Matallana, S. \& Segura, D. (2014). La loi Sarbanes-Oxley et sa besoin de implémentation dedans les institutions colombiennes comme une moyenne de solution aux cases de corruption dans les milliers financières. Activos 22, 195-228

\section{Résumé}

Le lois Sarbanes-Oxly mis en place aux États Unis et qui a actes législatifs en Colombie a une grande importance pour les entreprises que déroulent ses activités sociétaires dans le territoire, puis à travers de la même dirigent sa fonction et crédibilité auprès autres sociétés, comme les organisations qui cotisent à la bourse et que sont régulés par la Securities and Exchange Commission SEC.

Dans le corps de cette recherche on prendra l'histoire de la structuration de la loi Sarbanes-Oxley ses objectifs et importance dans le milieu nordaméricain. Aussi même on ferait une analyse des structures de régulation et les sanctions aux entités et aux professionnels que $\mathrm{n}$ arrivent pas à prévoir les divers responsabilités établis par cette loi

La finalité de cette travail d investigation est d'analyser si une moi avec les caractéristiques de la SOX est bien nécessaire au niveau colombien, car par les exemples claires du passé en matière de corruption dedans les entreprises ou les professionnels ne font pas ses devoirs à pleine responsabilité, peuvent adopter et faire la mise en place la législation SOX. Pour cela, il est nécessaire, en premier lieu, l’existence d'une commission chargée d'examiner le domaine de la formation pour les professionnels afin d'adopter et d'appliquer la loi; d'autre part, il est essentiel qu'il existe des sanctions applicables aux entités et aux professionnels, ce afin qu'aucun des scandales de corruption et, par conséquent, pas de fiabilité est perdu dans ces organes qui cotée sur la Bourse et qui son partie des différentes industries.

\section{Mots clés}

Loi Sarbanes-Oxley, la gouvernance d'entreprise, le contrôle interne, d'audit, de la corruption, de la comptabilité. 


\section{Introducción}

A comienzos del presente siglo se presentaron varios casos de desfalcos financieros en Estados Unidos, los cuales provocaron que la imagen tanto del contador público como del auditor se viera cuestionada por parte de la sociedad norteamericana, y que los aportes del ejercicio profesional al interés público perdieran credibilidad. En este contexto, el Congreso de los Estados Unidos sancionó en 2002 una ley cuyo fin es proteger al inversionista mediante la regulación de una normativa eficaz, en la que la auditoría, el control interno, el gobierno corporativo y la ética son principios esenciales en el seguimiento de los negocios financieros.

Esta ley, conocida como Sarbanes-Oxley Act, está orientada a regular la Securities and Exchange Commission [SEC], que es el principal organismo de fiscalización y normalización de los negocios bursátiles en el país. La Ley SOX fue el punto de partida para que los inversionistas recuperaran la confianza en el sistema financiero del país; también fue una forma de lograr que los directivos de las grandes corporaciones se concientizaran de la necesidad de proceder en la gestión de sus negocios teniendo en cuenta todos los requerimientos legales y éticos.

Así como ocurrió en Estados Unidos, en los últimos años se han evidenciado grandes escándalos de corrupción en Colombia, y el país ha tenido que superar desfalcos financieros de grandes empresas, los cuales han perjudicado a todo tipo de personas: inversionistas, contribuyentes, trabajadores, etc. Al igual que en el caso norteamericano, estos hechos hacen que la imagen del profesional contable, del auditor y de las mismas entidades de control se vea perjudicada, generando desconfianza por parte del público.

Por esta razón, el presente artículo pretende analizar tres aspectos básicos de la problemática: el primero parte de identificar si Colombia cuenta con un marco normativo similar al estructurado por la Ley Sarbanes-Oxley o si existe una iniciativa para adoptar esta norma, con el fin de controlar y regular las empresas, principalmente las que cotizan en la Bolsa de Valores 
de Colombia. El segundo aspecto parte de determinar qué empresas en Colombia aplican como parte de sus procesos de autorregulación los postulados de la Ley Sarbanes-Oxley en sus características y aspectos más relevantes. Por último, mediante un análisis de los casos de corrupción ocurridos hace unos años en Colombia, se pretende especificar si existía entonces una norma que regulara esos procesos financieros e identificar las entidades que los controlaban.

Este análisis se realiza con el fin de identificar la importancia de lo establecido por la Ley Sarbanes-Oxley en cuanto al control de las actividades financieras de una entidad, dando a conocer que sus principios y fundamentos son necesarios para un país que diariamente sufre episodios de corrupción.

Para darle solución a los tres aspectos expuestos anteriormente, el trabajo se divide en dos apartados. En el primero se identifica todo lo relacionado con la Ley Sarbanes-Oxley, sus antecedentes, historia, definición, objetivos e importancia. En el segundo, se abordan los aspectos relacionados con la normativa colombiana, se identifican las empresas que actualmente aplican esta ley y se realiza un análisis de los casos más relevantes de corrupción en Colombia, principalmente los que se han presentado en Bogotá.

\section{Aspecto teórico}

\section{Historia}

La Ley Sarbanes-Oxley fue aprobada el 24 de julio de 2002 en Estados Unidos, durante el Gobierno del presidente George W. Bush, luego de que el país pasara por una serie de escándalos financieros en los cuales se vieron involucradas empresas como Enron, Parmalat, World.com, entre otras.

Es así como en el año 2002, el senador demócrata Paul Spyros Sarbanes y el congresista Michael G. Oxley impulsaron esta nueva ley en Estados Unidos de América, encaminada a restablecer la confianza en los mercados de valores y reportes sobre la información financiera. (Mora y Salinas, 2008, p. 30) 
La figura del contador público en dichas empresas se vio afectada negativamente, por lo tanto, la desconfianza hacia estos profesionales se incrementaba cada vez más. La Ley Sarbanes-Oxley «no solo busca la seguridad y protección del inversionista, sino recuperar la credibilidad a la Auditoría, perdida debido a los continuos hechos que han contribuido a que la profesión se vea afectada de forma significativa» (Pardo, 2006, p. 27).

Basados en lo anterior, conceptos como gobierno corporativo, responsabilidad social empresarial, transparencia, entre otros, cobran importancia en esta ley, ya que para su efectivo desarrollo se tienen en cuenta distintas características, como las siguientes:

El gobierno corporativo en cualquier empresa cumple un papel de agrupación de normas y mecanismos que buscan la confiabilidad de las personas que participan de ella directa o indirectamente. Así mismo, aspectos como la responsabilidad social empresarial y la transparencia de los profesionales que se desempeñan en todas las entidades necesitan de un mínimo de características para el desempeño de sus actividades y la credibilidad de quienes participan en ellas, con el fin de mantener siempre al cliente en condiciones seguras de negocio.

En Estados Unidos, las entidades sometidas al cumplimiento de esta ley son aquellas empresas públicas registradas ante la Securities and Exchange Commission [SEC]. Como lo indica Moeller (2004): «La SEC tiene responsabilidad general de $\mathrm{SOA}^{4}$ y su auditoría $\mathrm{PCAOB}^{5}$ es la principal regla de fijación de la auditoría. Es por esta razón que la SEC ordena un informe financiero anual de control, que se denomina SEC 10-K» (p. 320).

4 Es el acto del Congreso de Estados Unidos, celebrado en julio de 2002, por el cual se regula la contabilidad pública. Establece nuevas normas para la gestión empresarial e introduce otros cambios para las auditorías de las empresas registradas en la SEC» (Moeller, 2004, p. 320).

5 «Es la Corporación General de la Junta de Contadores Públicos. Es una autoridad independiente responsable ante la SEC, que regula la profesión de contaduría pública en Estados Unidos y establece las normas de auditoría financiera» (Moeller, 2004, p. 320). 
Así mismo:

Todas las entidades registradas ante SEC deben crear un comité de auditoría [CA], el cual puede estar formado por consejeros o personas independientes; estos últimos no podrán devengar ingresos directos de la empresa, salvo los honorarios correspondientes por ser parte del comité. (Universidad Eafit, 2007, p. 3)

\section{¿Qué es la Ley Sarbanes-Oxley?}

Como lo describen Espejo Gavilán y Espejo Gavilán (2012), «el nombre de la ley se deriva de los apellidos de los dos principales patrocinadores: el diputado Michael G Oxley y el senador Paul S. Sarbanes» (p. 18).

Luego de una serie de escándalos presentados principalmente en Estados Unidos por empresas auditoras y la pérdida de confiabilidad hacia los profesionales contables, se buscó establecer la adopción de una norma que permitiera un control más profundo en las empresas, no solo en las auditoras, sino también en aquellas que participaran en el mercado de capitales ${ }^{6}$.

Morales (2005) afirma lo siguiente:

La ley influye, de manera muy significativa, entre otros, en los consejos de administración, en los directivos de esas empresas, en los bancos de inversión, en los analistas financieros y también de un modo importante en la actividad y regulación de los auditores de cuentas. (p. 109)

Los mercados americanos resultaron ser los principales afectados, y la visión interior y exterior era entonces cada vez más baja con respecto a la fiabilidad. Por lo tanto, se tomaron medidas para la recuperación de los inversionistas que formaran parte de dichos mercados.

6 «Es un tipo de mercado financiero en los que se ofrecen y demandan fondos o medios de financiación a medio y largo plazo» (Universidad EAFIT, 2007). 
Para Morales (2005), en esta ley se agrupan seis grandes áreas que intervienen en el desarrollo de los mercados financieros ${ }^{7}$ de Estado:

- Mejora de la calidad de la información pública y de sus detalles.

- Reforzamiento de responsabilidades del gobierno corporativo de las sociedades.

- Mejora de las conductas y los comportamientos éticos exigibles: mayores exigencias de responsabilidad en los temas de gestión indebida de información confidencial.

- Aumento de la supervisión de las actuaciones en los mercados cotizados.

- Incremento del régimen sancionador asociado a incumplimientos.

- Aumento de exigencia y presión sobre la independencia efectiva de los auditores.

Estas características permiten 1) la mejora de la información presentada por la empresa hacia el público en general; 2) que el auditor sea una pieza clave y su trabajo deba realizarse bajo supervisión, con el fin de que las responsabilidades que tiene ante la entidad se cumplan; 3 ) evitar las actuaciones ilegales por parte de los miembros de la entidad en relación con fraudes en la información; 4) la existencia de entidades externas que supervisen el desarrollo de las actividades del auditor en aspectos como estándares, ética, control, entre otros; 5) que las sanciones deban presentarse en cualquier existencia de manipulación de información mediante entidades encargadas de dichos procesos; y 6) que el aumento de exigencias hacia el

7 «Se componen de un mercado bursátil -o mercados de capitales a largo plazo(las Bolsas de cada país), de un mercado monetario de cambios -o mercados de capitales a corto y medio plazo-, de un mercado de tasas de interés, de un mercado de materias primas y de un mercado de productos derivados» (Zacharie, s,f, p. 1). 
auditor sea claro y que muestre sus responsabilidades y restricciones en su ámbito profesional.

\section{Importancia, estructura y objetivos de la ley}

La SOX ${ }^{8}$ cumple un papel importante en la auditoría y en el control interno de las empresas a las que va dirigida, lo que hace que los inversionistas no pierdan la credibilidad en sus actividades. Esto se debe a la rigidez de temas que contiene, como se verá a continuación.

Teniendo en cuenta la norma (Ley 107-204, 2002), aprobada por el Congreso de los Estados Unidos de América en julio 30 de 2002, la estructura de la Ley Sarbanes-Oxley se encuentra dividida en los siguientes títulos:

- Título 1. Junta de vigilancia de las compañías de contabilidad pública.

- Título 2. Independencia del auditor.

- Título 3. Responsabilidad de la compañía.

- Título 4. Revelaciones financieras más amplias.

- Título 5. Conflictos de interés del analista.

- Título 6. Recursos y autoridad de comisión.

- Título 7. Estudios e informes.

- Título 8. Responsabilidad de la compañía por fraude penal.

- Título 9. Responsabilidad penal de empleados.

8 «Es la abreviatura de la Ley Sarbanes-Oxley. Muchas publicaciones se refieren a la ley de esta manera. Como también se le llama SarbOx o SOA» (Moeller, 2004). 
- Título 10. Declaraciones juradas de impuestos de la compañía.

- Título 11. Fraude y responsabilidad de la compañía.

Cada uno de estos títulos está complementado con una serie de secciones que explican en profundidad las obligaciones y los derechos de las entidades, así como su regulación y efectos penales.

De acuerdo con Espejo Gavilán y Espejo Gavilán (2012), los objetivos principales de la ley son:

1. Monitorear la contabilidad.

2. Sancionar a los ejecutivos que cometen fraudes corporativos e incrementar el presupuesto para los auditores e investigadores de la SEC.

3. Centrarse en todo lo relacionado con la creación de procedimientos, documentándolos, controlándolos y comunicándolos, y velar por que las empresas mejoren su contabilidad mediante el uso de procedimientos financieros documentados.

4. Reglamentar a las firmas de auditoría y crear un comité de auditoría el cual puede estar formado por consejeros o personas independientes; estos últimos no podrán devengar ingresos directos de la empresa, salvo los honorarios por ser parte del comité.

\section{Ley Sarbanes-Oxley en Colombia}

En los últimos años, Colombia ha pasado por ciertos episodios negativos para la estabilidad del sistema financiero del país y de la economía. En aquellos sucesos predominaron el poder y la falta de ética de sus participantes, así como también faltó astucia por parte de los órganos de control (Junta Central de Contadores, Procuraduría General de la 
Nación, Contraloría General de la República, entre otros entes de control establecidos en el país).

Entre ellos se puede mencionar el carrusel de la contratación de Bogotá, el caso de Interbolsa, los desvíos de dineros de Salucoop, entre otros, que se analizarán más adelante. Estos casos de corrupción afectaron en gran medida la estabilidad financiera del país y la posibilidad de realizar negocios con empresas internacionales porque, debido a todos los sucesos negativos, no existía seguridad para estas entidades ni había una fuente de control eficaz que les proporcionara un respaldo económico en su inversión.

Para el cumplimiento de los objetivos planteados, a continuación se abordará la parte normativa que establece el Gobierno colombiano, con el fin de identificar si actualmente existe una norma similar a la Ley SarbanesOxley, que tenga como fundamento evitar que sigan ocurriendo casos de corrupción.

Se tendrán en cuenta la Ley 1474 del 12 de julio de 2011 (Estatuto Anticorrupción) y la Ley 43 de 1990 (Reglamento de la profesión de contador público). Se analizarán los aspectos más importantes de estas leyes.

Se comparará la Ley 1474 de 2011 con lo que indica la Ley SarbanesOxley y se identificará si hay relación entre estos documentos.

\section{Aspecto normativo en Colombia}

\section{Ley 1474 de julio de 2011 o Estatuto Anticorrupción}

El Gobierno nacional estableció en la Ley 1474 de julio 12 de 2011, también denominada Estatuto Anticorrupción, la necesidad de «fortalecer los mecanismos de prevención, investigación y sanción de actos de corrupción y la efectividad del control de la gestión pública» (Estatuto Anticorrupción, 2011). 
La Ley 1474 de 2011 se divide en ocho capítulos, de los cuales los más relacionados con el tema que se está tratando son los capítulos I, II y III, que tratan sobre las medidas administrativas, penales y disciplinarias para la lucha contra la corrupción pública y privada, así como también el capítulo $\mathrm{V}$, que establece los organismos especiales que controlan estos casos de corrupción.

Tomando como referencia lo establecido en la Ley 1474, se determina que esta normativa establece un criterio más formal ante aquellos episodios de corrupción, fijando medidas penales y disciplinarias para aquellas entidades y personas que incumplan los criterios éticos en el desarrollo de cualquier actividad financiera. También se resalta la responsabilidad del revisor fiscal y del auditor ante la información contable que se elabore, la cual debe estar libre de vicios y totalmente acorde con las normativas legales. Para su control eficaz, se designaron entidades públicas con el fin de verificar y estar pendiente del desarrollo de las actividades de estas entidades. En el Estatuto Anticorrupción se enfatiza especialmente en dos entidades: «la Comisión para la Moralización y la Comisión Nacional Ciudadana para la Lucha contra la Corrupción, las cuales tienen su antecedente en la Ley 190 de 1995» (Estatuto Anticorrupción, 2011).

Según el capítulo V (Organismos especiales para la lucha contra la corrupción), en el artículo 62 de la Ley 1474 de 2011 se establece la conformación de la Comisión Nacional para la Moralización, integrada por el presidente de la República, el ministro del Interior y de Justicia, el procurador general de la nación, el contralor general de la República, el auditor general de la República, entre otros.

También se debe tener en cuenta que el estatuto contempla medidas para mejorar la eficiencia y la eficacia del control fiscal en la lucha contra la corrupción. «Esta reforma pretende aumentar los índices de eficacia y con ello lograr la legitimidad del control fiscal frente a la ciudadanía» (Estatuto Anticorrupción, 2011, p. 15). 
Tabla 1. Comparaciones y diferencias entre la Ley Sarbanes-Oxley y la Ley 1474 de 2011

\section{Ley Sarbanes Oxley}

\section{Ley 1474 de 2011 o Estatuto Anticorrupción}

«Esta ley genera un marco de transparencia para las actividades y reportes financieros de las empresas que cotizan en la Bolsa, y le proporciona mayor certidumbre y confianza a inversionistas y al propio Estado» (Ley 107-204, 2002).
Se dictan normas orientadas a fortalecer los mecanismos de prevención, investigación y sanción de actos de corrupción y la efectividad del control de la gestión pública (Estatuto Anticorrupción, 2011).
«Se establece la Public Company Accounting Oversight Board para supervisar la auditoría de las compañías públicas» (Moeller, 2004).
Se crea la Comisión Nacional para la Moralización, presidida por el presidente de la Republica, y la Comisión Nacional Ciudadana de la Lucha contra la Corrupción.

El Estatuto Anticorrupción toma medidas drásticas para aquellas personas naturales, jurídicas o servidores públicos que se involucren en temas de corrupción, así como la fijación de inhabilidades para aquellas empresas y personas, y la cancelación de la matrícula de contadores o revisores fiscales cuando estos tengan conocimiento de estos casos de corrupción. la ley. También exige la creación de un comité de auditoría que mantenga ciertas reglas de independencia.

«El que a sabiendas altere, destruya, mutile, oculte, cubra, falsifique o realice asientos falsos en cualquier registro, documento u objeto tangible, con la intención de impedir, obstruir o influir en la investigación o en la administración inadecuada de cualquier asunto, será multado o encarcelado por no más de 20 años» (Ley 107-204, 2002).
El estatuto plantea una política de cero tolerancia a la corrupción, mediante ciertas medidas, con el fin de hacer un llamado preventivo. Entre ellas están la eliminación de los subrogados penales, la ampliación de los términos de prescripción para los delitos de corrupción y la extensión de la responsabilidad penal a las personas jurídicas, entre otras. 
Ley Sarbanes Oxley

La Ley Sarbanes-Oxley se enfoca principalmente en el inversionista, busca que se creen organizaciones que controlen a estos profesionales, con el fin de que la información que presenten esté libre de vicios y permita la toma de decisiones.
Ley 1474 de 2011 o

Estatuto Anticorrupción

El Estatuto Anticorrupción es una ley desarrollada objetivamente de acuerdo con las necesidades que tiene el país en los diferentes sectores, como es el caso de la salud.

Fuente: elaboración propia a partir de la Ley 1474 de 2011 (Estatuto Anticorrupción), Ley 107-204 de 2002 y aspectos teóricos de la Ley Sarbanes-Oxley.

De acuerdo con lo que se establece en el cuadro comparativo, en Colombia sí existe una norma que regula de alguna u otra forma el control en las empresas y que lucha contra los episodios de corrupción, estableciendo sanciones severas para que estos casos no sigan ocurriendo. Cabe aclarar que la aplicación de esta ley debe estar regulada por un organismo que cumpla sus funciones de forma específica, en el que los profesionales realicen su trabajo de manera adecuada para ofrecer un mayor control y credibilidad. La Ley Sarbanes-Oxley es más formal que el Estatuto Anticorrupción en varios aspectos, pero este último, mediante sanciones drásticas, da a conocer mecanismos de control con el fin de frenar los episodios de corrupción.

\section{Ley 43 de 1990}

En Colombia, la auditoría es regulada principalmente por la Ley 43 de 1990. En su artículo 7, sobre las normas de auditoría generalmente aceptadas, se hace referencia a lo siguiente:

«Las normas de auditoría generalmente aceptadas se relacionan con las cualidades profesionales del contador público, con el empleo de su buen juicio en la ejecución de su examen y en su informe referente al mismo». 
En dicho artículo se expresan tres normas fundamentales para la auditoría, a saber:

- Normas personales.

- Normas relativas a la ejecución del trabajo.

- Normas relativas a la ejecución de informes.

En estas normas, el profesional encargado de la auditoría es el eje, ya que su trabajo debe ser realizado con certeza, confiabilidad e imparcialidad en las decisiones que tome. Así mismo, en esta ley se tiene en cuenta el Código de Ética, que «hace referencia al revisor fiscal para establecer conceptos como certificación y dictamen de estados financieros y las responsabilidades de los administradores» (Pardo, 2006, p. 68).

El buen trabajo de los profesionales contables hace que la credibilidad en una empresa no se pierda, es por esto que el «subsistema profesional trata de evaluar lo correspondiente a cómo funciona la profesión en determinado país en aspectos como las asociaciones profesionales, el código de ética, el mercado de la auditoría, entre otros aspectos relevantes» (Patiño y Vásquez, 2013, p. 1024).

En la Ley-Sarbanes Oxley se exige un comité de auditoría, que es definido así: «Es un comité (o cuerpo equivalente) establecido por y dentro de una junta de directores de un emisor, con la finalidad de supervisar los procesos de reportaje de contabilidad y financiero del emisor, y auditorías del estado financiero del emisor» (Rozas, 2008, p. 35). Para esta ley es muy importante este comité porque asegura una mejor vigilancia de la información y del desarrollo de las actividades financieras.

Las leyes establecidas anteriormente permiten identificar la normativa que se tiene instaurada en Colombia con respecto al tema de corrupción. De la misma manera, se fijan las diferencias o similitudes que tienen estas leyes con la Ley Sarbanes-Oxley. 


\section{Entidades que aplican esta ley}

Según lo investigado, por ser una ley establecida en Estados Unidos, las empresas establecidas en Colombia que implementan en sus políticas esta ley actualmente son las entidades que cotizan en la Bolsa de New York, como las siguientes:

\section{Ecopetrol}

La reversión al Estado colombiano de la Concesión de Mares, el 25 de agosto de 1951, dio origen a la Empresa Colombiana de Petróleos (Ecopetrol, s. f.).

Ecopetrol emprendió actividades en la cadena del petróleo como una empresa industrial y comercial del Estado, encargada de administrar el recurso hidrocarburífero de la nación, y creció en la medida en que otras concesiones revirtieron e incorporó su operación (Ecopetrol, s. f.).

Desde el momento de su creación, el objeto social de Ecopetrol ha sido «el desarrollo, en Colombia o en el exterior, de actividades comerciales o industriales correspondientes o relacionadas con la exploración, explotación, refinación, transporte, almacenamiento, distribución y comercialización de hidrocarburos, sus derivados y productos» (Ecopetrol, s. f.). Durante todos estos años, este objeto social se ha cumplido, catalogándose actualmente como una de las mejores empresas en su campo. Y esto se ha logrado por el buen desarrollo y ejecución de sus objetivos.

La aplicación de los requerimientos de la Ley Sarbanes-Oxley por parte de Ecopetrol permite identificarla como una empresa sólida, ética en sus procesos administrativos y financieros, y con un control eficaz del desarrollo de su actividad financiera. También les hace posible a los inversionistas tener confiabilidad al momento de invertir en esta empresa.

Durante 2010, Ecopetrol continuó y consolidó su estrategia de cumplimiento de la Ley SOX 
(...) concentrando sus esfuerzos en el fortalecimiento de controles en aquellas áreas en donde se identificaron oportunidades de mejora, así como en el afianzamiento de la cultura de autocontrol y la sostenibilidad de las actividades de control interno sobre el reporte financiero. (Ecopetrol, s. f.).

Como se indica en la página web de Ecopetrol, al hablar del cumplimiento de esta ley se dice lo siguiente:

Así mismo, y de acuerdo con lo establecido por la Comisión de Valores de los Estados Unidos (SEC, por sus siglas en inglés), se emitió la certificación anual por parte del presidente y la vicepresidente corporativos de Finanzas, en cumplimiento con las disposiciones de las secciones 302 y 404 de la Ley SOX, en relación con los procedimientos y controles sobre revelaciones y el control interno sobre el reporte financiero con corte al 31 de diciembre de 2009, en la cual se declara una debilidad material sobre el proceso de reconciliación de estados financieros a estándar contable de los Estados Unidos. Esta debilidad no tiene incidencia en la confiabilidad de los resultados presentados a los inversionistas, los cuales presentan de manera razonable la situación financiera de la compañía, según fue certificado debidamente por el revisor fiscal.

\section{Grupo Bancolombia}

El Grupo Bancolombia ya contaba con políticas y procedimientos de control interno y riesgos mucho antes de que los escándalos financieros que dieron origen a la Ley Sarbanes-Oxley sucedieran.

El Grupo adoptó e implementó un esquema de administración de control interno para dar cumplimiento a esta ley, promulgada por el Congreso de los Estados Unidos en 2002, con el fin de recuperar la confianza de los inversionistas, aumentar la credibilidad de los accionistas en las sociedades e incrementar la transparencia de los estados financieros y la información contable. (Grupo Bancolombia, s. f.) 
Esta ley deben cumplirla todas las compañías listadas en la Bolsa de Nueva York a partir de 2006. (Grupo Bancolombia, s. f.)

Igualmente, la ley exige que un auditor independiente (revisoría fiscal) realice la evaluación del Sistema de Control Interno y emita una opinión sobre la efectividad del mismo. (Grupo Bancolombia, s. f.)

Teniendo en cuenta que la ley recomienda el uso del modelo de control interno «COSO» y que el Grupo Bancolombia tomó la decisión de adoptarlo, la evaluación del Sistema de Control Interno se realiza considerando las actividades desarrolladas para el cumplimiento de cada uno de los cinco componentes: ambiente de control, evaluación de riesgos, actividades de control, información y comunicación, y monitoreo» (Grupo Bancolombia, s. f.).

Como se puede observar, las empresas colombianas que han establecido la Ley Sarbanes-Oxley dentro de sus políticas son principalmente aquellas que cotizan en la Bolsa de New York.

También se deben tener en cuenta empresas cómo CEMEX y algunas firmas de auditoría, como Deloitte y Price Watherhouse Couper, que si bien no son empresas colombianas, llevan a cabo la prestación de sus servicios en nuestro país y dentro de sus políticas tienen implementada la Ley Sarbanes-Oxley.

\section{Empresa Cemex}

Es una empresa fundada en México en 1906. Se ha convertido en una empresa líder en su industria. La compañía llegó a Colombia en 1996, luego de la adquisición de «Cementos Samper, Cementos Diamante y Central de Mezclas, y hoy tiene presencia en más de veinte ciudades del país. Con sus soluciones se construyen importantes obras de infraestructura, vías y vivienda en todo el territorio» (Cemex, s. f.). 
Se toma como referencia esta empresa porque actualmente implementa la Ley Sarbanes-Oxley y porque cumple con cada uno de los requisitos. En sus palabras: «nos apegamos a la regla Fair Disclosure de la SEC, la cual prohíbe la divulgación selectiva de información aun cuando la regla no aplica a compañías extranjeras. Sin embargo, creemos firmemente que el seguir esta regla fortalece la integridad de los mercados de valores e incrementa la confianza de los inversionistas» (Cemex, s. f.).

Los pasos que ha implementado esta entidad para llevar a cabo la aplicación de la Ley Sarbanes-Oxley son los siguientes: certificación de reportes financieros por parte del director general y el director de finanzas; certificación interna, controles internos, Comité de Auditoría Independiente y Código de Ética.

\section{Casos de corrupción}

En Colombia han existido diferentes casos de corrupción en los que se ha evidenciado que las acciones de los organismos reguladores de la lucha contra la corrupción no son lo suficientemente rígidos para castigar los hechos presentados.

Algunos de los ejemplos recientes son los dados por Interbolsa, Saludcoop y el carrusel de la contratación en Bogotá por el Grupo Nule, casos en los que se observa que aspectos como la ética ${ }^{9}$ la responsabilidad social empresarial no fueron tenidos en cuenta por las empresas en cuestión; así mismo, en estos casos aparecen profesionales que no aplicaron sus conocimientos de forma correcta, sino que buscaron beneficios individuales.

La corrupción se puede entender como un acto desleal en el cual, quien recurre en este busca beneficios individuales que normalmente son económicos. Se pretende llevar un control de dichos actos mediante el

9 «Concierne, en griego, a la búsqueda de una buena "manera de ser" o la sabiduría de la acción» (Badiou, s. f.). 
Estatuto Anticorrupción de Colombia, con el fin de que sean castigados con medidas penales.

A continuación se muestra un acercamiento a tres de los casos de corrupción que reflejan las acciones reguladoras que necesitan más fuerza y rigidez en los castigos, y es en este punto donde se considera necesaria la aplicación de una ley como la Sarbanes-Oxley, mediante la cual se pueda adoptar una serie de medidas que de alguna forma logre mitigar o reducir desfalcos en las entidades colombianas.

\section{Interbolsa}

El sector financiero en términos generales ha tenido un comportamiento aceptable para evitar escenarios de crisis, en los últimos años se ha evidenciado un fenómeno mundial respecto a corporaciones financieras que entran en crisis para luego quebrarse. (Tautiva, 2014).

Interbolsa era una entidad dedicada a la administración de inversiones de grupos empresariales con el fin de brindar «un portafolio de productos con diferentes alternativas para distintos tipos de inversionista: carteras colectivas ${ }^{10} \mathrm{y}$ algunos de los principales fondos de capital privado ${ }^{11}{ }$ (Uribe, 2013).

Interbolsa, «entidad financiera perteneciente al mercado de valores, conocida en el sector como la empresa comisionista más grande del país» (Rodríguez, s. f.), luego de problemas de liquidez entró en quiebra, por lo que el gobierno optó por intervenirla «cuando la Superintendencia Financiera expidió la resolución para ordenar la toma de posesión de la

10 «Las carteras colectivas (generalmente conocidas como fondos) no son valores, sino productos a través de los cuales se reúnen recursos o dinero de diversas personas» (Portofolio.co, 2014).

11 «El capital de riesgo o capital privado es dinero aportado por inversionistas profesionales que invierten, con una visión de largo plazo, en el capital de empresas con gran potencial de crecimiento» (Portofolio.co, 2014). 
sociedad comisionista. La decisión se precipitó tras verificar que el día anterior Interbolsa incumplió el pago de una de sus obligaciones con el BBVA por la suma de 20000 millones de pesos» (Revista Semana, 2012).

El principal problema en este caso fue la especulación del aumento o disminución de las inversiones, lo que sucedió con los 'repos', que son títulos valores que se negocian en el mercado de valores con el fin de obtener liquidez. En este caso, los 'repos' pertenecientes a la compañía Fabricato, una entidad textilera a la cual Interbolsa le dio gran importancia, estaban en aumento, pero luego de un tiempo los inversionistas empezaron a desconfiar del precio de Fabricato. «El problema surgió cuando el mercado comenzó a desconfiar de que Fabricato pudiera valer eso. El 'repo' es un complejo engranaje con muchas piezas que depende básicamente de la credibilidad que los inversionistas le den a la evolución del precio. Si se llega a pensar que está inflado artificialmente, se bloquea la circulación del título y todo el andamiaje se viene abajo» (Revista Semana, 2012).

De acuerdo con la Revista Semana (2012), a partir de ese momento «a pesar de la restricción de liquidez, la comisionista venía operando normalmente. El presidente de la Bolsa de Valores de Colombia, Juan Pablo Córdoba, ratificó que Interbolsa ha venido honrando todas sus obligaciones. El superintendente financiero, Gerardo Hernández, aclaró que los problemas son de liquidez y no de solvencia, algo que sería mucho más grave. De hecho, la sociedad comisionista tiene un patrimonio de 139470 millones de pesos y su nivel de solvencia es 21,72 por ciento, por encima del 9 por ciento exigido por las autoridades».

Por esta situación, el gobierno decidió intervenir debido a que «un problema de liquidez temporal en una firma de la Bolsa se puede convertir en un hueco mayúsculo si no se para a tiempo, el gobierno prefirió adelantarse» (Revista Semana, 2012). 


\section{Saludcoop}

Es una entidad colombiana que brinda los servicios de salud y bienestar con el fin de generar la calidad humana e íntegra a todo tipo de personas. «Saludcoop se ha convertido en un grupo empresarial, y se encuentra entre las 20 empresas más grandes de Colombia, y es la primera en generación de empleo» (Saludcoop Eps, s. f.).

Dicha entidad fue intervenida debido a una serie de inconsistencias presentadas, entre ellas, la «ausencia de contratos con las EPS, deficiencias en el proceso de facturación, inconsistencias en su situación financiera y contable, reiteradas fallas en la prestación de los servicios, entre otras» (El Espectador.com, 2011). El Gobierno colombiano decidió intervenir esta entidad no solo por el hecho de la mala atención a los usuarios de este servicio: el mal manejo de los estados financieros hizo que se tomara dicha decisión.

El Estatuto Anticorrupción, emitido en 2011, da a conocer mecanismos de control que aseguran la prestación del servicio de salud, buscando implementar sanciones drásticas para las personas (representantes legales, contadores, auditores, directivos, entre otros) que atenten contra los recursos destinados principalmente al cumplimiento de este servicio dándoles otro uso para el beneficio propio.

Los organismos reguladores en este caso son el Ministerio de Salud, la Superintendencia de Salud y el Defensor del Pueblo, con el fin de que las actividades realizadas en dichas entidades tengan coherencia no solo en la atención de usuarios, sino en la revisión de la contabilidad y de los profesionales.

\section{Carrusel de la contratación en Bogotá}

El carrusel de la contratación en Bogotá, como fue denominado, es uno de los casos de corrupción más grande por el que no solo la capital, sino el país, ha tenido que atravesar. Los principales participantes en este caso 
son el Grupo Nule, conformado por los hermanos Manuel y Miguel Nule y su primo Guido Nule, además de una serie de integrantes que formaron parte de uno de los desfalcos más importantes del país.

El caso inició cuando los empresarios Nule perdieron la adjudicación de la Ruta del Sol, uno de los proyectos viales más importantes de Colombia, por lo que dichos empresarios, que ya venían con problemas económicos, fueron a la quiebra. "Para este joven grupo que desde hacía varios meses navegaba hacia el abismo por problemas de liquidez, cesación de pagos, elevado endeudamiento y malos manejos, quedarse con algún tramo de esta obra era cuestión de vida o muerte» (Revista Semana, 2010).

El Grupo Nule tuvo un gran momento cuando los negocios que ofrecían eran bien pagos, así como las obras de infraestructura en Colombia $\mathrm{y}$ en diferentes partes del mundo fueron importantes. «Con los negocios marchando viento en popa, los jóvenes Nule gastaban a igual ritmo. Tenían su propio jet, en el que viajaban con sus amigos por Colombia y fuera del país. Hacían gala de gran generosidad con sus invitados costeando de su bolsillo todos los gastos sin escatimar peso alguno» (Revista Semana, 2010).

Luego de su gran ascenso, se observaron deficiencias en los procesos de pago de deuda y retrasos en la construcción de proyectos viales. En ese punto entraron desfalcos financieros como «los escándalos por retraso en la construcción de la Troncal de TransMilenio en la calle 26, donde eran responsables del 70 por ciento de la obra. Ante este escándalo, el IDU ordenó que devolvieran 69000 millones de pesos, pagados como adelanto por estas obras, y se les ordenó ceder el contrato» (Revista Semana, 2010).

Como cualquier grupo empresarial, al existir cualquier deficiencia se empiezan a buscar métodos de solución, y esta no fue la excepción: «con los problemas hasta el cuello, los Nule intentaron en mayo de este año una salida. Le solicitaron a la Superintendencia de Sociedades la admisión a un proceso de reorganización empresarial contemplado en la ley, algo así 
como lo que en el pasado se llamó concordato, para parar el desangre en que estaban con embargos y demandas de docenas de acreedores histéricos» (Revista Semana, 2010).

Aunque las noticias no fueron alentadoras y la caída del grupo empresarial de los Nule se fue en picada, «la petición no solo fue negada, sino que la Superintendencia les cayó con todo el peso de la ley, pues encontró que las dos principales sociedades, MNV S.A. y Gas Kpital, tenían serias fallas» (Revista Semana, 2010).

Luego de que la noticia fue conocida por los colombianos, se expresaron opiniones de todo tipo y de toda clase: «algunos analistas que han visto de cerca la operación de este conglomerado dicen que hay mucho más de ineficiencia en el manejo que hacían en su operación, unida a un gasto excesivo y a un desorden administrativo enorme» (Revista Semana, 2010).

Así mismo, «algunos competidores que perdieron licitaciones frente a ellos se [atrevieron] a decir que como los sobornos fueron parte integral de la fórmula del triunfo, implicaban un sobrecosto adicional que agravaba los problemas de liquidez» (Revista Semana, 2010), lo que demuestra que lograr un ascenso tan rápido tiene sus conclusiones. A pesar de que en su momento fue un grupo muy importante en el ámbito nacional e internacional, pero por malas decisiones llegó a este punto.

No solo el Grupo Nule, sino diferentes participantes en dicho caso, «tomaron tajada»-como popularmente se dice-de las acciones realizadas y se vieron involucrados en dichos desfalcos, con los que al final perdieron los ciudadanos, quienes luego de todo este proceso llegaron a la conclusión de que las acciones tomadas en contra del Grupo y sus participantes no fueron las adecuadas, ya que las entidades que regularon dicho caso no se encontraron a la altura de medir el daño que estaban haciendo, razón por la se perdió mucho dinero y las obras de infraestructura tuvieron retrasos que no fueron planeados. 


\section{Responsabilidad de la auditoría en los escándalos financieros}

Desde el momento en que ocurre un caso de corrupción, como los descritos anteriormente, surgen ciertas preguntas: ¿dónde estaban los organismos de control?, ¿por qué los auditores internos o externos, o los revisores fiscales no fueron los que descubrieron el fraude?

Para responder esta pregunta, Ochoa, Zamarra y Guevara (2011) dan a conocer una descripción general de la auditoría, del proceso de la auditoría y de todos sus elementos esenciales, concluyendo que esto ocurre por deficiencias en el control a estos profesionales; también especifican que «la probabilidad de detectar errores generalmente es más alta que la de detectar fraude, ya que el fraude suele ser planeado para ocultar su existencia». Finalizan indicando que es necesario que el profesional esté muy preparado para llevar a cabo sus funciones y de la misma forma tenga un nivel crítico y ético bien fortalecido con el fin de que tome consciencia de sus actos.

Se enfatiza en este aspecto porque la Ley Sarbanes-Oxley exige un comité de auditoría y un organismo de control para que regule a los profesionales con el fin de evitar una información que no refleje la realidad.

\section{Conclusión}

Así como le ocurrió a Estados Unidos, Colombia, en los últimos años, fue partícipe de varios escándalos financieros que afectaron en gran medida la situación financiera y económica del país. Al ser conscientes de esta realidad, como estudiantes, futuros profesionales y líderes de un país, la presente investigación abordó tres objetivos claros relacionados con esa problemática teniendo en cuenta la Ley Sarbanes-Oxley.

A continuación se desarrollará un análisis general de cada objetivo establecido y se finalizará argumentando si la Ley Sarbanes-Oxley es 
necesaria en las entidades colombianas como control para que no se sigan presentando más casos de corrupción.

En Colombia, el gobierno y los entes de control fueron conscientes de las grandes consecuencias que produjo cada uno de los eventos de corrupción, por lo tanto, establecieron una ley y un estatuto que tuvieran como objetivo luchar contra esta gran problemática por medio de la implementación de sanciones drásticas y penales a los partícipes de estos casos. En cambio, la Ley Sarbanes-Oxley fue creada como un respaldo principalmente para el inversionista, que le brinda seguridad mediante un control eficiente de la información financiera y de los profesionales que la elaboran. Esta ley principalmente enfatiza en las empresas que cotizan en la Bolsa.

Las dos leyes tienen en común la búsqueda de información confiable y oportuna para la toma de decisiones y el buen desarrollo ético en las actividades financieras mediante la descripción general de las funciones del revisor fiscal y del auditor, teniendo como base la ética en el desarrollo de su profesión. Se puede decir que esta normativa (Ley 1474 de 2011) previene en parte la corrupción, pero a diferencia de la Ley Sarbanes-Oxley, hace falta un control más rígido, que controle constantemente las funciones de los contadores y auditores en sus actividades diarias, que les exija reportes diarios con el objetivo de verificar que sus procedimientos se estén realizando correctamente con base en la normativa legal contable vigente.

Teniendo como pilar las fuentes consultadas, se puede decir que la Ley Sarbanes-Oxley no está establecida en Colombia en la actualidad como una ley de prevención ni de control ante los casos de corrupción; tampoco se tiene la iniciativa de adoptarla. En cambio, como normativas que tratan el tema de corrupción en Colombia se encuentran la Ley 1474 de 2011 (Estatuto Anticorrupción) y la Ley 43 de 1990, entre otras.

Sin embargo, como lo indica el Grupo Bancolombia (s. f.) en sus políticas y mecanismos de control interno, «la Ley Sarbanes-Oxley exige a las entidades inscritas en la Bolsa de Valores de Nueva York (NYSE) el establecer, mantener y valorar la efectividad del Sistema de Control Interno para la 
presentación de reportes financieros». Es decir, las empresas colombianas que coticen en la Bolsa de New York deben, dentro de sus políticas, implementar la Ley Sarbanes-Oxley, la cual les garantiza mayor control y respaldo a sus inversionistas.

Entre las empresas colombianas que dentro de sus políticas establecen la Ley Sarbanes-Oxley se destacan Ecopetrol, Bancolombia y las demás empresas nacionales que cotizan en la Bolsa de New York. En general son muy pocas, porque en Colombia sobresalen las pyme. Sería interesante que el Gobierno colombiano les diera más atribuciones y responsabilidades a los entes de control del país para que ejercieran una supervisión más rigurosa, no solamente a las empresas públicas o a las que cotizan en la Bolsa, sino a todo tipo de entidades, según el sector de la actividad económica de cada una de ellas.

Al hablar de los casos de corrupción, como se observó a lo largo del trabajo, se pretende que estos sucesos, en Colombia y en el mundo, sean vistos como aspectos necesarios para fortalecer el sistema de regulación del país, pero que a la vez se tome consciencia de que se deben evitar de la mejor forma, mediante una normativa exigente y controladora, capaz de combatir estos episodios. Así mismo, se reconoce la importancia de que la educación de los profesionales debe ser estricta, basada en valores y sentido crítico, con el fin de que la credibilidad de estos hacia las entidades no se pierda.

Casos como los de Enron, World.com, Parmalat y los sucedidos en los últimos años en Colombia son una base que muestra la delicadeza del asunto en lo que a desfalcos y sanciones se refiere; así mismo, se observa que al final de todo esto los perjudicados son los ciudadanos y los inversionistas.

Como se analizó en cada caso, la normativa de control establecida en Colombia era muy débil y los organismos de control encargados de la auditoría y revisoría fiscal de estas entidades no llevaron a cabo sus funciones de forma correcta. 
Finalmente, la Ley Sarbanes-Oxley es una gran alternativa para el control de la información financiera, pues le brinda al inversionista seguridad y confianza. Sería muy interesante que las grandes y medianas empresas colombianas aplicaran esta ley en sus políticas; pero hay que tener en cuenta que esta ley solamente es para las empresas que cotizan en la Bolsa, así como también deben considerarse los altos costos de su implementación. Por esta razón, se concluye que para evitar que casos de corrupción sigan dándose en Colombia, la solución consiste en que las entidades tanto públicas como privadas tomen consciencia del buen manejo de sus recursos, y el gobierno establezca un control más eficiente a las empresas (grandes y medianas) y cree entidades u organismos que vigilen y controlen constantemente al profesional contable, al auditor, al representante legal y demás directivos importantes de una organización, ya que sobre ellos recae la responsabilidad de cumplir y hacer cumplir estas leyes. Es decir, es fundamental no dejar que estas personas busquen su beneficio propio y se apoderen de los recursos de otras personas, acrecentando su patrimonio a expensas de los demás.

Para lograr esto, es necesario crear consciencia en todos los entes reguladores tanto públicos como privados, en las entidades y principalmente en los futuros profesionales en contaduría pública, auditoría y revisoría fiscal, para que fortalezcan la imagen de la profesión por medio del desarrollo eficaz de sus funciones, tomando como base la ética y el cumplimiento de todas las normas, ya que de nosotros depende cambiar la historia de nuestro país. 


\section{Referencias bibliográficas}

Badiou, A. (2004). La ética: ensayo sobre la conciencia del mal. México: Herder.

Banco de la República. (27 de enero de 2011). Boletín de Indicadores Económicos.

Recuperado de http://www.banrep.gov.co/sites/default/files/paginas/bie.pdf.

Barrientos, M. y Sarmiento, D. (2012). Normas internacionales de información finan-

ciera NIIF-IFRS. Ejemplos, ejercicios y casos prácticos comentados. Bogotá: CEF.

Cámara de Comercio de Medellín. (2011). Normas Internacionales de Información Financiera NIC-NIIF. Recuperado de http://goo.gl/zo551W.

Cemex. (s.f.). La Ley Sarbanes-Oxley. Recuperado de http://www.cemex.com/ES/ Inversionistas/LeySarbanesOxley.aspx.

Cemex. (s.f.). Acerca de Cemex. Recuperado de http://www.cemexcolombia.com/ NuestraEmpresa/AcercaCemex.aspx.

Comisión Europea. (2006). La nueva definición de pyme. Guía del usuario y ejemplo de declaración. España: Publicaciones de Empresa e Industria.

DANE. (26 de junio de 2013). Encuesta de microestablecimientos. Panel de firmas 2012. Bogotá: Oficina de Prensa DANE.

Decreto 410 de 1971. Código de Comercio de Colombia, Diario Oficial n. 33339 de junio 16 (1971).

Decreto 2706 de 2012. Por el cual se reglamenta la Ley 1314 de 2009 sobre el marco técnico normativo de información financiera para microempresas, Diario Oficial n. ${ }^{\circ} 48657$ de diciembre 28 (2012).

Departamento Administrativo Nacional de Estadística [DANE]. (2005). Censo general. Bogotá: DANE.

Departamento Nacional de Planeación [DNP]. (2007). Documento Conpes 3484. Política nacional para la transformación productiva y la promoción de las micro, pequeñas medianas empresas: un esfuerzo público-privado. Bogotá: Consejo Nacional de Política Económica y Social.

Ecopetrol. (s.f.). Cumplimiento de la Ley Sarbanes-Oxley (SOX). Recuperado de http://goo.gl/MKzgOY.

Ecopetrol. (s. f.). Nuestra historia. Recuperado de http://goo.gl/Ei7O5C.

El Tiempo. (31 de mayo de 1991). Microempresas dominan la economía colombiana. El Tiempo. Recuperado de http://goo.gl/y9yCxk. 
Espejo Gavilán, N. J., y Espejo Gavilán, A. O. (2012). Estudio comparativo de la Ley Sarbanes-Oxley con el nuevo Estatuto Anticorrupción (Ley 1474 de 2011) y aplicabilidad del aseguramiento de la información en Colombia (Trabajo de grado). Universidad Militar Nueva Granada, Bogotá. Recuperado http://goo. $\mathrm{gl} / 5 \mathrm{v} 4 \mathrm{Tsm}$.

Fundación IFRS. (2009). Material de formación para las Pymes. Módulo 35: Transición a las NIIF para las Pymes. Londres: IFRS.

Giraldo, B. H. (2007). Estadística de la microempresa en Colombia. Análisis comparativo 1990-2005. Bogotá: Corporación para el Desarrollo de la Microempresa y Observatorio Colombiano de las Microempresas (OCM).

Gonzáles, I. (2003). Contabilidad internacional. México: McGraw-Hill.

Grupo Bancolombia. (s. f.). Políticas y mecanismos de control interno Grupo Bancolombia. Recuperado de http://goo.gl/KIMUyM.

Hernández, R. M. (2006). La perspectiva sociológica de los actores empresariales. En Enrique de la Garza (Coord.), Tratado latinoamericano de sociología (pp. 209-227). México: Anthropos y Universidad Autónoma Metropolitana.

IFRS. (2008). NIIF 1. Adopción por primera vez de las normas internacionales de información financiera. Recuperado de http://plancontable2007.com/niif-nic/ niif-normas-internacionales-de-informacion-financiera/niif-1.html.

IFRS. (27 de mayo de 2014). Marco conceptual para la presentación de información financiera. Recuperado de eifrs.iasb.org: http://eifrs.iasb.org/eifrs/bnstandards/es/2012/Framework.pdf

Kaplan, G. \& Johnson, A. (1988). La contabilidad de costes: auge y caída de la contabilidad de gestión. Barcelona: Plaza y Janés Editores S. A.

Ley 222 de 1995. Por la cual se modifica el libro II del Código de Comercio, se expide un nuevo régimen de procesos concursales y se dictan otras disposiciones, Congreso de Colombia, Diario Oficial n. ${ }^{\circ} 42156$ del 20 de diciembre (1995).

Ley 107-204. Ley Sabarnes-Oxley de 2002. Por la cual se establecen nuevas regulaciones de control interno, auditoría y sanciones, 15 U. S. C. $\$ 7201$, Congreso de Estados Unidos (2002).

Ley 590 de 2000. Por la cual se dictan disposiciones para promover el desarrollo de las micro, pequeñas y medianas empresas, Congreso de Colombia, Diario Oficial n. ${ }^{\circ} 44078$ del 12 de julio (2000). 
Ley 905 de 2004. Por medio de la cual se modifica la Ley 590 de 2000 sobre promoción del desarrollo de la micro, pequeña y mediana empresa colombiana y se dictan otras disposiciones, Congreso de Colombia, Diario Oficial n. ${ }^{\circ}$ 45628 de agosto 2 (2004).

Ley 1314 de 2009. Por la cual se regulan los principios y normas de contabilidad e información financiera y de aseguramiento de información aceptados en Colombia, se señalan las autoridades competentes, el procedimiento para su expedición y se determinan las entidades responsables de vigilar su cumplimiento, Congreso de Colombia, Diario Oficial n. 47409 de julio 13 (2009).

Ley 1450 de 2011. Por la cual se expide el Plan Nacional de Desarrollo, 2010-2014, Congreso de Colombia, Diario Oficial n. 48102 de junio 16 (2011).

Ley1474 de 2011. Por la cual se dictan normas orientadas a fortalecer los mecanismos de prevención, investigación y sanción de actos de corrupción y la efectividad del control de la gestión pública, Congreso de la República, Congreso de la República, Diario Oficial n. ${ }^{\circ} 48128$ de julio 12 (2011).

Manay, G. M. (2012). Las micro, pequeñas y medianas empresas (mipymes) y su participación en el desarrollo social crecimiento económico de América Latina. Perú: Centro de Estudios Latinoamericanos (CESLA).

Moeller, R. (2004). Sarbanes-Oxley and the New Internal Auditing Rules. New Jersey: John Wiley y Sons, Inc.

Montoya, C. C. (2011). El racionamiento de crédito a las microempresas en Colombia. Un estudio de los tipos de racionamiento. Coyuntura Económica: Investigación Económica y Social, 41(1), 225-255.

Mora Pedraza, L. y Salinas Daza, W. (2008). Análisis de la Ley Sarbanes-Oxley como herramienta para el mejoramiento de la calidad de la información financiera en Colombia (Trabajo de grado). Universidad de San Buenaventura, Bogotá. Recuperado de http://goo.gl/YCcn9C.

Morales, J. D. (2005). La ley Sarbanes-Oxley y la auditoría. Partida Doble, 169, 104109. Recuperado de http://pdfs.wke.es/5/3/4/4/pd0000015344.pdf.

Ochoa, L., Zamarra, J. y Guevara, J. (2011). ¿Cuál ha sido la responsabilidad de la auditoría en los escándalos financieros? Contaduría Universidad de Antioquia, 58-59, 117-145.

Pardo, K. (2006). Análisis de la Ley Sarbanes-Oxley respecto a la independencia que debe tener el contador al analizar y dictaminar los informes financieros 
(Trabajo de grado). Universidad de La Salle, Bogotá. Recuperado de http:// goo.gl/sjX8x7.

Patiño-Jacinto, R. A. y Vásquez-Quevedo, N. (2013). Las características de los subsistemas contables de Colombia y México ante la convergencia a normas internacionales de información financiera. Cuadernos de Contabilidad, 14(36), 1009-1043. Recuperado de http://goo.gl/cw2dpe.

Pérez, S. (7 de diciembre de 2012). Empresas del sector real impulsaron las emisiones de renta fija en el mercado. La República. Recuperado de http://goo. gl/LzhDMF.

Portafolio.co. (5 de mayo de 2011). Así operan las carteras colectivas en el mercado. Portafolio. Recuperado de http://goo.gl/aFR64i.

Quintero, H. G. y Peña, L. (2012). Guía de entendimiento del proyecto de norma de información financiera para las microempresas - Consejo Técnico de la Contaduría Pública (Trabajo de grado). Pontifica Universidad Javeriana, Bogotá. Recuperado de http://goo.gl/m7UvJp.

Ramírez, M. C. (4 de octubre de 2013). Culpables, jueces y verdugos en la caída de la primera comisionista de Bolsa. La República. Recuperado de http://goo. gl/SQIoAO.

Redacción Vivir. (14 de marzo de 2011). Nuevo golpe a Saludcoop. El Espectador. Recuperado de http://goo.gl/69ETFI.

Revista Semana. (septiembre, 2010). La caída del Grupo Nule. Semana. Recuperado de http://goo.gl/1nQCss.

Revista Semana. (noviembre, 2012). La verdad sobre la comisionista Interbolsa. Semana. Recuperado de http://goo.gl/QembVj.

Rozas, A. E. (2008). El comité de auditoría. Quipukamayoc, 15(29), 33-42. Recuperado de http://goo.gl/71nH3F.

Saludcoop Eps. (s.f.). Quiénes somos. Recuperado de http://goo.gl/6CaoOT.

Superintendencia de Sociedades. (2012). Comportamiento de los grupos empresariales del sector real de la economía. Informe. Bogotá: Superintendencia de Sociedades-Grupo de Estudios Económicos y Financieros.

Tautiva, C. C. (2014). Una aproximación al caso Interbolsa (Trabajo de grado). Universidad del Rosario, Bogotá.

Universidad EAFIT. (21 de septiembre de 2007). Mercado de capitales. Consultorio Contable. Área Contable. Boletín 63. Recuperado de http://goo.gl/5OG9xn. 
Universidad EAFIT. (29 de marzo de 2007). Ley Sarbanes-Oxley. Consultorio Contable. Área Auditoría. Boletín 49. Consultorio Contable Área de Auditoría y Control. http://goo.gl/RQsJnb.

Universidad EAFIT. (29 de marzo de 2007). Sarbanes Oxley. Consultorio Contable. Área Auditoría. Boletín 17. http://goo.gl/GkGOue.

Uribe, E. M. (3 de junio de 2013). Qué es Interbolsa y sus funciones en Colombia. Recuperado de http://emiliomartinuribe.com/que-es-interbolsa -y-su-funciones-en-colombia/

Zacharie, A. (s.f). Los mercados financieros. Recuperado de http://www.rebelion. org/docs/5713.pdf 